\title{
IDEOLOGÍAS POLÍTICAS Y ADAPTACIÓN PARTIDARIA: EL CASO DEL MLN-TUPAMAROS (1985-2009)*
}

\author{
Political Ideologies and Party Adaptation: \\ The Case of MLN-Tupamaros (1985-2009)
}

\author{
ADOLFO GARCÉ \\ Universidad de la República, Uruguay
}

\begin{abstract}
RESUMEN
El artículo reconstruye el proceso de adaptación del MLN-Tupamaros a la legalidad y a la competencia electoral desde la restauración de la democracia en Uruguay (marzo de 1985) hasta la elección de José Mujica, fundador del MLN-T y candidato del Frente Amplio, como presidente de Uruguay (noviembre de 2005). Esta trayectoria constituye un caso especialmente notable de éxito de un proceso de adaptación partidaria. El argumento central es que la sorprendente capacidad de adaptación de esta organización debe explicarse, fundamentalmente, por algunos rasgos de su matriz ideológica que se distinguió, en la izquierda uruguaya, por su pragmatismo.
\end{abstract}

Palabras clave: Adaptación partidaria, ideología, pragmatismo, tupamaros, Uruguay.

\begin{abstract}
The article rebuilds the process of adaptation of the MLN-Tupamaros to legality and electoral competition since the restoration of democracy in Uruguay (March 1985) and the election of José Mujica, founder of the MLN-T and candidate of the Frente Amplio, as president of Uruguay (November 2005). This path is a particularly remarkable case of successful party adaptation. The central argument is that the remarkable adaptability of this organization must be explained mainly by some features of its ideological matrix which is distinguished, in the Uruguayan left, for his pragmatism.
\end{abstract}

Key words: Party adaptation, ideology, pragmatism, tupamaros, Uruguay.

* Una primera versión de este texto fue presentada en el Área Temática 11 "Movilización sociopolítica, violencia y revolución en América Latina", coordinada por Eduardo Rey Tristán y Alberto Martín, del XIV Encuentro de Latinoamericanistas Españoles. Congreso Internacional 1810-2010: 200 años de Iberoamérica (16 al 18 de setiembre, Universidad de Santiago de Compostela). Muchos colegas brindaron generosamente insumos para la elaboración de este artículo. Quisiera agradecer especialmente a Flavia Freidenberg, Andrés Malamud, Alberto Martín, Salvador Martí, Guillermo Mira, Juan Andrés Moraes, Pierre Ostiguy, Vicente Palermo, Eduardo Rey Tristán, José Rilla, Jaime Yaffé y a los referís anónimos de RCP. 


\section{EL MLN-T COMO CASO EXITOSO DE ADAPTACIÓN PARTIDARIA}

El Movimiento de Liberación Nacional Tupamaros (MLN-T) surgió a medidos de los años 60 en el marco de la onda expansiva provocada por la revolución cubana. En 1972 su estructura militar fue desmantelada y sus principales dirigentes y cientos de militantes encarcelados (Labrousse, 2009). En junio de 1973 la disolución del Parlamento marcó el hito fundamental en la instalación de un régimen autoritario que duró hasta 1984. Al restablecerse la democracia, en 1985, los presos políticos fueron amnistiados. Muchos ex tupamaros no se reincorporaron a la militancia. Otros prefirieron debatir con sus viejos compañeros qué hacer en las nuevas circunstancias. En la mayoría de ellos la experiencia de la dictadura había desencadenado un proceso de aprendizaje político acerca del valor de las garantías institucionales de la poliarquía (Arrarás, 1988). Finalmente, prevaleció la estrategia de reorganizar la estructura político-militar pero actuando en la legalidad. Simultáneamente tramitó el ingreso al Frente Amplio, la coalición de izquierdas fundada en 1971. A partir del ingreso al FA en 1989 el MLN-T fue aprendiendo a manejarse cada vez mejor en la arena electoral. Para maximizar su desempeño electoral y luchar contra el "reformismo" dentro de la izquierda, a comienzos de 1989, crearon el Movimiento de Participación Popular (MPP). Poco a poco comprendieron que si querían ampliar su electorado también ellos debían moderar su discurso. Conquistaron diputados en 1994, senadores en 1999, y aportaron un importante caudal de votos al triunfo de Tabaré Vázquez en 2004 (Yaffé, 2005; Garcé, 2006). En el 2009, José Mujica, uno de los fundadores de la guerrilla tupamara, logró ser electo candidato a la presidencia por el FA derrotando en la elección primaria a Danilo Astori, el candidato auspiciado por el presidente Tabaré Vázquez. En la elección de octubre las listas que respondían al liderazgo de Mujica obtuvieron 7 de los 16 senadores electos por el FA. En el balotaje, un mes después, Mujica derrotó por amplio margen al ex presidente Luis Alberto Lacalle (Altman, 2010; Buquet y Johnson, 2010; Garcé, 2010). Mujica, finalmente, asumió como presidente de Uruguay el $1^{\circ} \mathrm{de}$ marzo de 2005. Cuatro de los trece ministros militaron en el MLN-T. ${ }^{1}$

Mujica logró ser electo presidente de Uruguay sin dejar de reivindicar su pasado guerrillero. En términos comparados, la evolución del MLN-T probablemente represente un caso extremo de éxito en la adaptación de una organización guerrillera a la competencia electoral. En este artículo se argumentará que, aunque otros factores coadyuvaron, no es posible explicar la trayectoria de la organización sin entender la peculiar estructura de la ideología política tupamara. Las ideas no son solamente un parche, un argumento ad hoc al que cabe acudir cuando fracasan los enfoques centrados en instituciones o intereses. Las ideas pueden explicar algunos procesos políticos mejor que la dinámica de los intereses y que las estructuras organizativas. En este sentido, en términos teóricos, este artículo debe ser colocado en el contexto del ideational turn reivindicado temprana y elocuentemente por Mark Blyth (1997), brillantemente reseñado y reorganizado recientemente en Schmidt (2008).

1 Eduardo Bonomi (Ministro del Interior), Ricardo Ehrlich (Ministro de Educación), Daniel Olesker (Ministro de Salud Pública) y Luis Rosadilla (Ministro de Defensa). 


\subsection{Ideología y adaptación partidaria}

No todos los partidos logran adaptarse del mismo modo a los cambios en el entorno y a la competencia electoral. La literatura sobre el tema, especialmente a partir de Kitschelt (1994), Panebianco (1995) y Levitsky (2005), insiste en que esto depende de los procesos políticos intrapartidarios, es decir, de la dinámica de los liderazgos y de las coaliciones internas. Aunque también toman en cuenta otras variables, los enfoques más divulgados priorizan el estudio de la dimensión organizacional de los partidos como variable independiente de la capacidad de adaptación. Partiendo del supuesto de que el factor liderazgo es clave, argumentan que éste no se ejerce en el vacío sino al interior de determinadas reglas de juego fijadas por la organización. ${ }^{2}$

En el contexto de una literatura en la que predominan los enfoques centrados en las restricciones y oportunidades generadas por las estructuras organizativas, algunos autores han puesto de manifiesto la influencia de factores ideológicos en el proceso de adaptación partidaria. Quien, a mediados de los 90, más se aproximó a considerar a la ideología como variable independiente fue Herbert Kitschelt. Según este autor, para explicar de qué modo concreto algunos partidos llevaron adelante procesos de adaptación estratégica subóptimos, es necesario tomar en cuenta las tradiciones discursivas de estos partidos y del sistema político en el que están inmersos: "I will propose that political traditions identifying acceptable arguments and ideas shape the internal discurse in political parties and limit the range of strategic choices when parties are faced with new challenges". (1994: 254). Según Kitschelt las ideas no explican por qué un partido dado termina adoptando una estrategia electoral subóptima sino por qué la estrategia escogida tuvo cierta dirección y no otra:

"The fact that parties diverge from electoral strategic rationality is explicable in terms of organizational mechanisms of interest articulation and aggregation within parties (...), yet not the content of the political appeals that diverge from party's electoral rationality. For this reason, the cases of British Labour Party and the Swedish social democrats have been particularly instructive. In both instances (...) only a logic of ideas shows how and in which way that divergence was accomplished" (278-279).

La reconstrucción del proceso del MLN-T que se propone en este artículo procura desarrollar este aspecto concreto del enfoque teórico de Kitschelt. Con las ideologías ocurre lo mismo que con las estructuras organizativas: algunas de ellas facilitan más que otras los procesos de adaptación partidaria. Para entender de qué manera la ideología puede afectar el proceso de adaptación hay que distinguir dos dimensiones. La primera dimensión es el contenido, esto es, el stock específico de valores, creencias y posiciones sobre políticas. Tal como explicara Kitschelt, el contenido de la ideología limita las alternativas de cambio del partido. La segunda dimensión es el tipo de ideología. Es perfectamente posible que ideologías con contenidos similares tengan un impacto muy diferente sobre la capacidad de adaptación de un partido dado. Algunas ideologías

2 Numerosos autores han seguido, en mayor o menor medida, la huella abierta por estos dos autores, aplicándola a casos de partidos de América Latina: Samuels (2004) al Partido de los Trabajadores de Brasil, Yaffé (2005) al Frente Amplio de Uruguay y por Santiuste Cué (2001) y Martí (2010) al del Frente Sandinista de Liberación Nacional de Nicaragua. 
facilitan los cambios estratégicos, otras los dificultan. El proceso del MLN-T ilustra claramente, como se verá a continuación, de qué modo concreto las características de la ideología pueden favorecer la adaptación partidaria.

\subsection{La ideología tupamara}

Según la acertada expresión de Carlos Real de Azúa, el MLN-T no fue una guerrilla sino un "movimiento político con armas". ${ }^{3}$ Como regla general, los tupamaros calculaban muy cuidadosamente el impacto potencial de sus acciones en términos de popularidad. No deja de ser paradójico: los tupamaros no supieron organizar una guerrilla invencible, ni acelerar el proceso revolucionario, ni fabricar un atajo hacia la insurrección popular. Ni siquiera pudieron participar en la Huelga General de 1973. Pero, haciendo propaganda armada, comunicando sus ideas mediante acciones arriesgadas e ingeniosas, utilizando pocas palabras pero perfectamente comprensibles por el uruguayo medio, comunicándose por medio de símbolos, conectando hábilmente su lucha con las principales tradiciones revolucionarias del país y, muy especialmente, con el artiguismo, demostraron que sí sabían cómo hacer política (Panizza, 1990). En el fondo, lo mejor que tuvieron como guerrilleros fue lo que tenían de políticos: picardía, intuición, capacidad de comunicación. Es muy importante, para el argumento general, retener esta idea. Muchos, como Raúl Sendic y José Mujica, habían hecho política sin armas, antes de desencantarse del camino electoral y de encandilarse con la teoría del foco. Sendic militó durante muchos años en el Partido Socialista de Uruguay. Mujica, inició su carrera política en el Partido Nacional, más específicamente en la fracción orientada por Luis Alberto de Herrera. En este sentido, el caso del MLN-T lleva agua para el molino de Allison (2010) acerca de la importancia de la experiencia política previa como variable explicativa en el éxito de los procesos de transición de los movimientos armados a maquinarias electorales.

La principal diferencia entre los tupamaros y el resto de la izquierda no tiene que ver con los objetivos políticos sino con los procedimientos. Mientras que la izquierda comunista y socialista asignaba una gran importancia a la elaboración teórica, la actuación parlamentaria y la lucha electoral, los tupamaros pensaban que el ejemplo cubano demostraba que "las condiciones subjetivas se crean luchando" y que la lucha armada no sólo era la única vía a la revolución en América Latina sino el "principal método de lucha", al que deberían subordinarse todos los demás. Para los tupamaros, la práctica revolucionaria era más importante que la teoría revolucionaria. De hecho, lo dijeron y lo escribieron reiteradamente: "la teoría surge de la práctica" y "no hay mejor teoría revolucionaria que la que surge de las revoluciones hechas". ${ }^{4}$

3 Ver: Carlos Real de Azúa, Partidos, política y poder en el Uruguay (1971- Coyuntura y pronóstico), Facultad de Humanidades y Ciencias, Universidad de la República, Montevideo, 1988, pp. 102-104.

4 Esta frase todavía puede leerse en la página Web del MLN-T. Ver: http:/ / www.chasque.net/mlnweb/portada. htm. Visitado por última vez el 7-02-2011. 
En torno a esta visión se reunieron revolucionarios que provenían de muy distintas tradiciones políticas y teóricas, y que abrevaban en distintas fuentes doctrinarias: marxismo, ${ }^{5}$ leninismo, anarquismo, liberalismo, nacionalismo (Lessa, 2004; Rey Tristán, 2005; Garcé, 2006). La heterogeneidad ideológica se reflejó tanto en sus documentos como en sus prácticas. Los escasos documentos elaborados por el MLN-T se han caracterizado por su eclecticismo. Nunca fueron "puros" desde el punto de vista ideológico o teórico porque, tradicionalmente, fueron resultado de transacciones entre las diversas corrientes existentes dentro de la organización. La heterogeneidad ideológica se manifestaba también en las prácticas políticas de los tupamaros. A lo largo de la historia del MLN-T ha sido habitual que diversas organizaciones llevaran adelante, al mismo tiempo, estrategias políticas diferentes. Esto fue posible porque el MLN-T, históricamente, reconoció amplias zonas de autonomía a sus distintas estructuras. La descentralización, en el fondo, derivaba del pragmatismo. Para los tupamaros no era posible determinar a priori cuál es la teoría más correcta. Era necesario poner en práctica las concepciones existentes para ir construyendo, a partir de la práctica, la teoría revolucionaria. En suma, en teoría, de acuerdo a los sucesivos reglamentos, la organización se estructuraba sobre la base del centralismo-democrático. Sin embargo, en los hechos, pese a la leyenda construida en torno a algunas de sus acciones armadas más espectaculares, el MLN-T fue una "organización desorganizada" (permítaseme el juego de palabras que parafrasea a Levitsky), una guerrilla invertebrada, mutante, camaleónica, capaz de tomar muchos rumbos de acción diferentes a la vez.

Otro rasgo ideológico muy característico del MLN-T es el peso del componente nacionalista. El nacionalismo se manifestó de modos diversos. En primer lugar, en la jerarquía atribuida a la "liberación nacional" dentro de la estrategia revolucionaria del movimiento. Comunistas, socialistas y tupamaros coincidían, con terceristas y dependentistas, en la necesidad de la ruptura de los lazos de dependencia con el imperialismo. Pero unos ponían el acento en la lucha por "salvar al obrero de la explotación capitalista", los otros, en la lucha por "rescatar la patria del imperialismo". En segundo lugar, el nacionalismo se expresó en reiterada vocación de "independencia política". El MLN-T insistió, a lo largo de toda su historia, entre otras razones para diferenciarse de los comunistas, en que él sí era absolutamente independiente de cualquier organización internacional. El MLN-T llevó su independencia de criterio al extremo, adoptando posiciones distintas de las de algunos de sus referentes más destacados como Regis Debray o Ernesto Guevara. El nacionalismo tuvo una manifestación más: para el MLN-T no sólo había que pensar como uruguayos; además, los revolucionarios debían poder comunicarse en el lenguaje de los uruguayos. Toda la izquierda uruguaya, desde mediados de los años cincuenta, venía experimentando un proceso que los especialistas han caracterizado como de "tradicionalización" o "nacionalización" (Caetano y Rilla, 1995: 48-51; Lanzaro, 2004: 33; Yaffé, 2005: 119-123). Para poder "hablar en uruguayo", los tupamaros solían presentarse frente a la opinión pública como los continuadores de la tradición de lucha revolucionaria en el país, desde

5 Aunque no comparto su interpretación, me parece importante mencionar que según el excelente trabajo de Gatto (2004) el peso del marxismo y del leninismo en la ideología tupamara ha sido subestimado. 
Artigas y su lucha por la independencia nacional en las primeras décadas del siglo XIX hasta Aparicio Saravia y sus revoluciones contra los gobiernos colorados (fines del siglo XIX y comienzos del siglo XX).

Finalmente, la textura ideológica del MLN-T se caracterizó por mostrar una inconfundible raíz libertaria. La huella del anarquismo en la cultura política uruguaya no ha sido suficientemente estudiada. A cuenta de esa deuda, es necesario señalar al menos dos puntos importantes de afinidad entre el anarquismo y la tradición política del MLN-T. El primero es la elección de la propaganda armada como método de creación de conciencia (Rey Tristán, 2005). ${ }^{6}$ El segundo es la resistencia a las jerarquías, dentro y fuera de la organización. Los tupamaros jamás reconocieron líderes. En palabras de Marenales: “Al MLN-T no hay nadie que lo mande. Ni Raúl Sendic. Esto es un colectivo verdadero. Somos fanáticos del colectivo" (Garcé, 2006: 39).

\section{CINCO MOMENTOS EN LA TRAYECTORIA RECIENTE DEL MLN-T}

El 14 de marzo de 1985 fueron liberados los últimos presos políticos. Entre ellos, recuperó la libertad el pequeño grupo de dirigentes del MLN-T (los "rehenes") que, desde 1973 a comienzos de 1984, habían sido encarcelados en condiciones especialmente brutales. ${ }^{7}$ De inmediato, los "rehenes" brindaron una conferencia de prensa en la que reafirmaron la posición adelantada por Sendic durante sus últimos meses de cautiverio: teniendo en cuenta las "nuevas circunstancias" políticas abiertas por el fin del régimen autoritario, los tupamaros se comprometían a actuar en la legalidad. Efectivamente, desde 1985 en adelante el MLN-T no ha vuelto a organizar un foco guerrillero. Por el contrario, la organización se ha ido comprometiendo crecientemente con la lucha electoral y aprendiendo a jugar cada vez mejor con estas reglas de juego. Sin embargo, el proceso no fue sencillo. Hubo, como en organizaciones similares, un debate estratégico interno muy intenso que desembocó, a menudo, en rupturas importantes. En este capítulo se repasa la trayectoria de la organización durante cada uno de los cinco gobiernos que precedieron el ascenso de José Mujica a la presidencia.

\subsection{Sanguinetti I (1985-1989): la inserción en la legalidad}

La decisión de deponer las armas representaba un punto de inflexión impactante en la historia del MLN-T. Para los tupamaros, desde el comienzo, lucha armada y política revolucionaria habían constituido dos conceptos inseparables, dos caras de la misma moneda. Esta definición, simple y tremenda, estaba en los códigos ideológicos genéticos

6 Según Rey Tristán, "serían en 1876 anarquistas italianos los que realizasen una declaración proclamando la propaganda mediante la acción [...]: creían que el hecho insurreccional destinado a afirmar los principios socialistas mediante la acción es el medio de propaganda más efectivo y el único que sin engañar ni corromper a las masas puede penetrar hasta las capas sociales más profundas [...]. Esta estrategia política nacida del anarquismo, presente en todo el movimiento revolucionario de la época, fue la adoptada por el MLN-T" (2005: 181).

7 Entre ellos había fundadores del MLN-T como Raúl Sendic, Eleuterio Fernández Huidobro, Julio Marenales, Jorge Manera y José Mujica y otros dirigentes como Jorge Zabalza, Mauricio Rosencof y Henry Engler. 
del MLN-T. Desde luego, hubo polémicas. Pero la inmensa mayoría de los tupamaros estuvieron de acuerdo con Sendic en cuanto a que las circunstancias imponían la inserción en la legalidad. Sin embargo, discreparon con él sobre otro tema fundamental: según Sendic, el MLN-T no debía ser reorganizado. Para el viejo líder, los tupamaros debían, desde luego, seguir militando por hacer avanzar la perspectiva revolucionaria pero estaban llamados a inventar nuevas herramientas organizativas. En particular, propuso construir amplios movimientos de masas en torno a los problemas más graves y urgentes (en esa línea fue que, sin esperar el apoyo formal de sus compañeros, organizó el "Movimiento por la Tierra y contra la Pobreza"). También lanzó la idea de articular un "Frente Grande", esto eso, una gran alianza política y social de fuerzas progresistas en torno a un programa "mínimo" de soluciones a los principales problemas del país (plan para combatir la pobreza, aumento de salarios y jubilaciones, redistribución de la tierra, no pago de la deuda externa, etc.).

Contra la opinión de su principal referente público, durante 1985 los tupamaros avanzaron rápidamente en su reorganización. A fin de año celebraron la III Convención Nacional (20, 21 y 22 de diciembre). Esta convención, además de elegir el Comité Central y de ratificar algunas decisiones adoptadas por otras instancias organizativas del MLN-T en los meses previos (como la solicitud de ingreso al Frente Amplio o la decisión de actuar legalmente), debatió intensamente las razones de la derrota militar de 1972. Se presentaron 43 planteos de autocrítica. ${ }^{8}$ La convención no adoptó una decisión definitiva sobre el tema. De todos modos, quedaron claros algunos acuerdos importantes. En primer lugar, los casi dos mil participantes en estos debates no cuestionaron haber tomado las armas durante la década del sesenta. En segundo lugar, la mayoría opinó que el MLN-T había subestimado el trabajo de elaboración teórica: "por reacción frente al teoricismo de la izquierda, caímos en el error diametralmente opuesto: descuidamos los problemas teóricos y la actividad formativa". ${ }^{9}$ En tercer lugar, había cierto acuerdo en que, hacia comienzos de los setenta, la organización no logró incorporar al pueblo a la lucha revolucionaria.

Más allá de las coincidencias explicitadas durante los debates de la III Convención, subsistían diferencias importantes entre los tupamaros. Aun corriendo el riesgo de simplificar demasiado, es muy útil construir dos tipos ideales polares con identidades ideológicas y referentes políticos claros: en un extremo los "frentegrandistas", en el otro los "proletarios". Los frentegrandistas ${ }^{10}$ se identificaban con los nuevos planteos de Raúl Sendic. Para ellos, la coyuntura democrática era una oportunidad para hacer avanzar

8 En realidad, este tema venía siendo discutido desde fines de 1972. Ya desde entonces se perfilaban dos grandes interpretaciones de la derrota, que volvieron a expresarse en la III Convención. Un enfoque, defendido fundamentalmente por los dirigentes históricos, consideraba que la derrota del MLN-T se había debido a errores en el terreno de la estrategia político-militar. El otro ponía el acento en aspectos ideológicos. De acuerdo a esta otra visión, la derrota del MLN-T habría sido la consecuencia de insuficiencias teóricas e ideológicas, derivadas, a su vez, de la inconveniente composición de clase de la organización y sus direcciones.

9 MLN-T, III Convención, Tesis ideológica, Autocrítica, 1985.

10 Algunos de los más connotados frentegrandistas fueron Alba Antúnez, Edmundo Canalda, María Elena Curbelo, Diego Piccardo, Washington Rodríguez Belletti, y los dirigentes del Frente Juvenil. Contaban con el respaldo del propio Raúl Sendic. 
el grado de conciencia, organización y lucha popular. El MLN-T debía sacar el máximo provecho de las instituciones políticas y multiplicar la zona de contacto entre el MLN-T y el pueblo. Por eso mismo, pusieron mucha energía en la construcción de movimientos sociales y mucha imaginación en el desarrollo de los medios de comunicación (el quincenario Mate Amargo y la Radio Panamericana). En el polo opuesto a los frentegrandistas estaban los proletarios. ${ }^{11} \mathrm{Al}$ debatir la autocrítica, habían tendido a coincidir en explicar la derrota de la organización por sus "carencias" ideológicas y teóricas, y por la baja presencia de obreros. Para ellos era fundamental que el MLN-T abrazara el marxismo-leninismo y jerarquizara el papel de la clase obrera como actor central del proceso revolucionario. En el fondo, proletarios y frentegrandistas se disputaban la tradición tupamara. La corriente conformada en torno a Sendic era la mejor expresión de la enorme ambición política del MLN-T inicial, siempre orientado a encontrar el atajo hacia el poder y la revolución; de su sorprendente capacidad para romper con los moldes teóricos del resto de la izquierda; de su desconfianza respecto al leninismo; de su tolerancia a la diversidad teórica e ideológica dentro de la organización revolucionaria. La corriente de los proletarios era la expresión de otra faceta básica de la identidad del MLN-T inicial: su convicción en el papel decisivo e irremplazable de la lucha armada, de su predisposición hacia los aspectos militares de la lucha revolucionaria, de su vocación por transgredir los límites institucionales del Estado y de la legalidad.

Había una tercera corriente que también pretendía ser la más auténtica expresión de la tradición tupamara. No se sentían identificados con ninguno de los dos polos. Compartían algunas de las ideas de unos y de otros. En general, intentaban articularlas. Salvo Sendic, que claramente lideraba una de las corrientes, y el trío integrado por Jorge Zabalza, Julio Marenales y Andrés Cultelli, que aparecían como los principales referentes de la otra, la mayoría de los "rehenes" y de los dirigentes históricos del MLN-T formaban parte de este subconjunto. Además, se inclinaba hacia esta suerte de punto medio la mayoría de los militantes tupamaros que habían participado en la reconstrucción de las estructuras políticas del FA. ${ }^{12}$

En este período ninguna de las corrientes mencionadas logró predominar sobre las otras. Los documentos aprobados por IV Convención del MLN-T, celebrada en junio de 1987, fueron, como era tradicional en la organización, una transacción entre las distintas visiones coexistentes. El MLN-T reafirmó su pasado guerrillero y su vocación revolucionaria, no descartó ninguna "vía a la revolución", pero, al mismo tiempo, reclamó el fortalecimiento del Frente Amplio y convocó a un Frente Grande.

11 Algunos de los más destacados integrantes de esta corriente eran Nora Castro, Andrés Cultelli, Mario De León, Irma Leites, Hugo Leytón, Julio Marenales, Roberto Villanueva y Jorge Zabalza.

12 Los principales referentes de esta posición intermedia y esencialmente articuladora eran Eleuterio Fernández Huidobro y José Mujica. Junto a ellos, hay que mencionar también a Jorge Quartino, Eduardo León Duter y Eduardo Bonomi. Como no tuvieron una identidad claramente definida, no corresponde hablar de una corriente de opinión propiamente dicha. Pese a su heterogeneidad, jugaron un papel muy importante en la articulación de las diferencias entre los dos polos y, por ende, fueron decisivos en la conservación de la unidad de la organización. Expresaron, en este sentido, otro de los aspectos más destacados de la matriz original del MLN-T: su capacidad para hacer convivir diferentes ideologías y enfoques políticos. 


\subsection{Lacalle (1990-1994): preparándose para el "nuevo 73"}

La muerte de Raúl Sendic a fines de abril de 1989 marcó un punto de inflexión en la evolución del MLN-T. Mientras que durante los primeros años de la reorganización las distintas corrientes internas habían logrado coexistir de un modo relativamente pacífico, reconociéndose, como había sido tradicional, grandes zonas de mutua autonomía y distribuyéndose espacios de poder, durante esta segunda fase los proletarios desencadenaron exitosamente una ofensiva por el control de la organización. Uno de los primeros bastiones de poder político interno perdidos por los frentegrandistas fue el control de los medios de comunicación que, a fines de 1989, pasaron a ser controlados por los proletarios. Pero el momento clave fue la V Convención, realizada entre junio y julio de 1990. Allí los proletarios lograron imponer su visión en los aspectos más importantes. En la Tesis sobre Estrategia aprobada, podía leerse: "Si nuestro análisis de la crisis es correcto y la acumulación de fuerzas se produce, el Uruguay marcha hacia la confrontación y el Golpe de Estado". En sintonía con esta visión, los proletarios insistieron en que la organización debía prepararse para resistir el, inexorable, "nuevo 73 " ${ }^{13}$ El MLN-T debía volver a estructurarse como una Organización Político-Militar capaz de vertebrar, llegado el momento, la "insurrección popular". Quienes enfrentaron, en esta coyuntura, el giro "militarista" pagaron un precio caro. Algunos frentegrandistas fueron expulsados. Otros, como la inmensa mayoría del Frente Juvenil, se vieron obligados a abandonar la organización. El grupo intermedio, fiel a su tradición de privilegiar la búsqueda de la unidad, intentó articular las diferencias. Fracasaron. Pero los principales referentes históricos (Eleuterio Fernández Huidobro, Julio Marenales, José Mujica y Jorge Zabalza) permanecieron en la estructura.

La hegemonía de los proletarios agudizó la tensión, clásica, entre el MLN-T y el FA. Los tupamaros enfrentaron algunas de las decisiones adoptadas por Tabaré Vázquez desde el gobierno departamental de Montevideo. Pero el momento de máxima tensión se vivió en julio de 1994, cuando el MLN-T se opuso a la creación del Encuentro Progresista, una alianza electoral entre el FA y pequeños grupos políticos de distinto origen (colorados, blancos, democristianos, etc.). Los nuevos socios del FA exigían dos condiciones: (i) que Vázquez fuera el candidato a la presidencia y (ii) que el programa de gobierno se moderara (Yaffé, 2005: 170-176). Esta perspectiva era resistida, aunque no exactamente por las mismas razones, por Líber Seregni, Danilo Astori, militantes del Partido Comunista y el MPP. La mayoría del MPP estaba francamente en contra de la "macrocoalición". En el II Congreso del MPP realizado en diciembre de 1993, el único grupo que se pronunció a favor de esta nueva política de alianzas fue el Partido por la Victoria del Pueblo (PVP). Por esa razón, este partido optó por abandonar el MPP (junio 1994).

13 Preparando el "nuevo 73, durante estos años, de acuerdo a diversos testimonios, grupos del MLN-T hicieron entrenamiento militar básico dentro y fuera del país. El MLN-T recibió apoyo financiero de organizaciones similares como el Movimiento Revolucionario Tupac Amaru peruano y del Movimiento Todos por la Patria argentino. También se realizaron actividades de "finanzas" al estilo de los años 60. Leicht (2007: 163-164, 182). 
El predominio del "horizonte insurreccional" dentro del MLN-T no impidió que la organización decidiera autorizar a sus integrantes a integrar listas electorales a cargos nacionales y departamentales. En 1989, luego de extensas discusiones, había prevalecido en el MLN-T la posición contraria. Hacia 1993, en cambio, había consenso entre los tupamaros en que era posible compatibilizar el "horizonte insurreccional" con la presencia en instituciones del "régimen burgués". Uno de los argumentos fundamentales para adoptar esta decisión fue que, luego de la elección de 1989, el MLN-T había evaluado que el magro resultado electoral obtenido por el MPP debía explicarse, al menos parcialmente, por la ausencia de tupamaros en las listas.

Pese a la inclusión de reconocidas figuras del MLN-T en las listas del MPP, el desempeño electoral de esta fracción en 1994 fue, nuevamente, mediocre. El MPP obtuvo casi la misma cantidad de votos que en la elección anterior (aproximadamente 45.000). Pero su participación relativa disminuyó porque la votación de la izquierda, gracias a la candidatura presidencial de Tabaré Vázquez y a la formación del Encuentro Progresista, experimentó un incremento notable: desde el 20\% al 30\% del electorado. El Partido Colorado, ganador de esa elección, obtuvo $32 \%$ de los votos.

Es posible que una de las razones de la mala votación del MPP haya sido su protagonismo en el hecho más violento registrado durante los últimos 20 años en Uruguay: la "masacre de Jacinto Vera". En mayo de 1992 fueron arrestados trece ciudadanos españoles de origen vasco por poseer documentación falsa. España solicitó la extradición de ocho de ellos por pertenecer a la ETA. La justicia uruguaya aprobó la extradición de tres. Entre 1993 y 1994 los vascos hicieron huelgas de hambre para obtener asilo político. El 19 de agosto, viendo que la salud de los detenidos corría peligro, el gobierno optó por internarlos en un hospital montevideano (el Hospital Filtro). El 24 de agosto de 1994 llegó un avión de España para concretar la extradición. A instancias del MLN-T, alentados por Mujica y Fernández Huidobro desde diversos medios de comunicación, los alrededores del Hospital Filtro se fueron cubriendo de manifestantes. La situación se fue tensando. Para trasladar a los vascos el gobierno no vaciló en reprimir la manifestación. Según testimonios de la época, del lado de los manifestantes hubo piedras y hasta algún disparo (Labrousse, 2009: 207-210).${ }^{14}$ El balance fue impactante: 80 heridos y un manifestante muerto por heridas de bala. El 26 de agosto, el gobierno del Presidente Lacalle decretó la clausura de la Radio Panamericana, apelando a vicios formales en el contrato.

\subsection{Sanguinetti II (1995-1999): la seducción de las urnas}

No es posible saber si, de no haber ocurrido el episodio del Hospital Filtro, el FA hubiera ganado la elección. En todo caso, lo que está fuera de discusión es que el MLN-T pagó 
un precio político muy alto dentro del FA por haber impulsado la manifestación. Los tupamaros fueron severamente cuestionados por Líber Seregni, líder histórico del FA, y por Tabaré Vázquez, la estrella en ascenso. El resultado electoral mostró con claridad meridiana que era perfectamente posible que el FA, más temprano que tarde, terminara ganando las elecciones nacionales. La dirección del MLN-T, habituada a los cambios bruscos de timón en función de las circunstancias, modificó el rumbo. A partir de 1995 se convirtió en un aliado fundamental de Tabaré Vázquez y de quienes venían, desde hacía muchos años, propiciando la moderación programática del FA para facilitar la victoria electoral.

De todas maneras, la nueva estrategia generó controversias dentro del MLN-T y del MPP. En 1995 Zabalza abandonó el MLN-T y se convirtió, desde el MPP, en una figura central de la minicoalición que intentó impedir que el MPP avalara la nueva estrategia. En todo caso, los sucesos de 1994 y 1995 ponen de manifiesto, una vez más, la flexibilidad táctica de la organización. En 1994, el MLN-T había quedado del lado de Seregni y Astori en la defensa de la tradición frenteamplista, enfrentando el proyecto "centrista" de Vázquez. Un año después, la misma dirección que se había batido duramente contra la creación del Encuentro Progresista, no vaciló en emprender un camino nuevo, acercándose a su antiguo adversario, Tabaré Vázquez.

Si el resultado de la elección de 1994 había mostrado lo poco que le faltaba a la izquierda uruguaya para acceder al gobierno, a partir de su ingreso a la Cámara de Diputados, Mujica fue descubriendo su propio potencial político. Muy rápidamente logró conquistar un espacio muy importante en los medios de comunicación. El interés de los periodistas (y de la opinión pública) por Mujica no sólo tenía que ver con su condición de ex guerrillero. En realidad, todo en él llamaba la atención. En primer lugar, su apariencia: Mujica nunca se puso un traje o una corbata para ir al Parlamento. Por el contrario, siguió vistiéndose de la misma forma que antes de ser parlamentario, con ropa vieja, ajada, como la de los trabajadores más pobres. En segundo lugar, su estilo de vida: Mujica siguió viviendo en la misma chacra, realmente muy humilde, en la que se había instalado desde que salió de la cárcel, situada en un barrio pobre de la periferia de Montevideo. En tercer lugar, su lenguaje: en el polo opuesto del político tradicional (que, o era abogado o trataba de aparentarlo), lejos de los códigos de comunicación de los dirigentes de la izquierda tradicional (universitarios, intelectuales), el líder tupamaro utilizó sistemáticamente un lenguaje sencillo, popular, directo. La eficacia del lenguaje de Mujica no derivaba solamente del tipo de palabras o de expresiones utilizadas para expresar sus ideas sino, además, de la forma de pronunciarlas. En cuarto lugar, también llamó la atención de los comunicadores y del público por sus ideas. Desde el comienzo, fue evidente que ese dirigente no sólo era diferente a los demás por su aspecto, su forma de vivir o de hablar. Además expresaba conceptos poco habituales en políticos de izquierda y realmente inesperados en un ex guerrillero.

El ingreso de Mujica al Parlamento le dio al viejo guión frentegrandista el emisor y la caja de resonancia que le estaban faltando. El ex guerrillero se fue transformando en una figura habitual en los medios de comunicación, y en un protagonista clave del debate público. 
En setiembre de 1997 la fracción de Mujica (denominada, en ese momento, Corriente de Izquierda $)^{15}$ fue la segunda más votada en la elección interna del Frente Amplio. Entre fines de 1998 y principios de 1999 se realizó el IV Congreso del MPP. La dirección del MLN-T, encabezada por Eleuterio Fernández Huidobro, José Mujica y Eduardo Bonomi, logró imponer dentro del MPP la estrategia de moderación programática y de apoyo al liderazgo de Vázquez que se había esbozado a comienzos de 1995. Sarthou y Zabalza, figuras emblemáticas de los primeros años del MPP, abandonaron el MPP. Pese a la escisión, el MPP obtuvo una excelente votación: en octubre de 1999 Mujica fue electo senador. Había nacido lo que periodistas y analistas denominaron el "fenómeno Mujica".

Al mismo tiempo que Mujica se las ingeniaba para ir tendiendo puentes hacia la opinión pública, Fernández Huidobro se ocupó de tejer una alianza con sectores de la policía y de las Fuerzas Armadas (Leicht, 2007: 179-181). En setiembre de 1995, en ocasión de la visita de Fidel Castro a Uruguay, el "aparato" del MLN-T fue convocado por los servicios de inteligencia de la policía para "mantener la seguridad del visitante" (Garcé, 2006: 145). El segundo capítulo de estos contactos se registró durante 1996 y, otra vez, en torno a visitantes extranjeros. Los reyes de España, Juan Carlos y Sofía, habían sido invitados por Sanguinetti a visitar Uruguay. El servicio secreto español, con los antecedentes del "Filtro" sobre la mesa, temían que el MLN-T pudiera facilitar un atentado de la ETA contra los visitantes. Para disipar toda duda, los servicios secretos terminaron reuniéndose con dirigentes tupamaros y militares uruguayos. En el marco de esos contactos, surgió la posibilidad de que el MLN-T facilitara las negociaciones entre el gobierno español y la ETA. Fernández Huidobro viajó a España en 1998 para colaborar en estas negociaciones (Labrousse, 2009: 224). A partir de estos hechos, los contactos entre dirigentes tupamaros y militares se profundizaron (Labrousse, 2009:223-225). El objetivo de esta política era llevar tranquilidad a las FF.AA. ante la perspectiva de un gobierno de izquierda.

\subsection{Batlle (2000-2004): que la pradera no se incendie}

La presidencia de Batlle estuvo signada por la peor crisis económica en, al menos, cien años. Entre 1999 (último año de la presidencia de Sanguinetti) y 2002, el PBI per cápita cayó 18,7\%. La desocupación trepó a casi 20\%. La pobreza, que había caído durante la primera década de gobiernos democráticos, creció de 15\% a 31\% (De Armas, 2005). En una situación económica y social mucho peor a la de los años 60 (tiempos de estanflación) el MLN-Tupamaros adoptó una estrategia completamente distinta. En lugar de intentar "incendiar la pradera" (como antes del quiebre de la democracia), aprovechó la oportunidad para moderar todavía más su discurso. En agosto de 2001, la IX Convención del MLN-T hizo pública una declaración convocando a conformar "una gran correntada popular de

15 El MLN-T después del alejamiento del PVP y de la mala votación del MPP en 1994 buscó nuevas alianzas electorales. Esto fructificó en la conformación de la Corriente de Izquierda. Convergieron en este proyecto, además del MPP, pequeños grupos de izquierda radical: Partido Socialista de los Trabajadores -trotsquista-, Movimiento 26 de Marzo (liderados por Raúl Sendic-hijo), Movimiento 20 de Mayo (batllistas de izquierda), Izquierda Frenteamplista Independiente, Corriente de Unidad Frenteamplista, Unión Popular (Enrique Errohijo), Tendencia Marxista (Julio Louis), etc. En realidad, otra vez, los tupamaros quedaron aliados al ala más de izquierda del FA. 
carácter nacional, es decir, ancha y patriótica", con el objetivo de resistir el "letal proceso de desmantelamiento y descapitalización" del "aparato productivo".

José Mujica, cumpliendo con este mandato, dedicó grandes esfuerzos a tejer la alianza entre el MPP y nuevos sectores sociales. En primer lugar, tendió un puente hacia los pequeños y medianos productores rurales. Paulatinamente, logró conquistar la confianza de diversas corporaciones vinculadas a la actividad agropecuaria como la Federación Rural o la Asociación de Cultivadores de Arroz. El acercamiento con estos sectores empresariales culminó, a fines de 2001, en la experiencia de la "Concertación para el Crecimiento". Ésta se integró con 18 gremiales empresariales que reivindicaron la necesidad de un cambio profundo en la política económica del gobierno y un "gran acuerdo productivo nacional". El 16 de abril de 2002, la "pinza social" a favor de un cambio en la política económica terminó de cerrarse: la "Concertación para el Crecimiento" y el PIT-CNT convocaron juntos a una movilización que culminó en la lectura de una proclama en la que empresarios y trabajadores coincidían en el reclamo del cambio en la política económica.

La política adoptada desde 1995 (cooperación con Vázquez, discurso conciliador hacia los militares, moderación programática en aras de la "reconstrucción nacional") le permitió al MPP ampliar todavía más su caudal electoral. En la elección de octubre de 2004, la lista al Senado encabezada por José Mujica fue la más votada dentro del FA (30\% de los votos del FA, 15\% del total de votantes). En 1963 los tupamaros habían querido ser la chispa que incendiara la pradera. Cuarenta años después, en una situación económica y social mucho peor, tomaron el camino opuesto: aprovecharon la oportunidad de la crisis para moderar todavía más su discurso y para prepararse para ser una fuerza decisiva en el inminente gobierno frenteamplista. Como veremos en seguida, a pesar de la votación obtenida por la fracción de Mujica, Vázquez prefirió otros aliados. Los tupamaros no perdieron la paciencia y se tomaron la revancha.

\subsection{Vázquez (2005-2009): la batalla por la sucesión}

Tabaré Vázquez logró ser el sucesor de Seregni en el liderazgo del FA porque logró interpretar mejor que Danilo Astori las preferencias de la mayoría de los frenteamplistas. ${ }^{16}$ En 1994, Vázquez fue, por primera vez, candidato a la presidencia por la izquierda uruguaya. A principios de 1996, en el contexto del debate sobre la estrategia ante la reforma electoral, logró convertirse en el líder del FA desplazando a Seregni (que renunció a la presidencia del FA) y a Danilo Astori (que fue reelecto senador, esta vez creando su propia fracción, Asamblea Uruguay). Una vez que logró consolidarse como líder del FA impulsó una estrategia sofisticada que le permitió consolidar su hegemonía en la interna y facilitar el crecimiento del FA. Impulsó, al mismo tiempo, por un lado, la moderación del programa

16 En este sentido, fue fundamental su decisión de no apoyar la reforma constitucional promovida por Sanguinetti durante su segunda presidencia, con el apoyo del Partido Nacional. Tanto Líber Seregni como Danilo Astori apoyaron la reforma propuesta porque incorporaba la regla de la candidatura única por partido, una vieja aspiración de la izquierda uruguaya. Tabaré Vázquez, en cambio, se opuso, argumentando que el precio que se pagaba era demasiado alto: la incorporación de la segunda vuelta que, claramente, apuntaba a dificultar el triunfo del FA (Yaffé 2005: 176-179). 
del FA (profundizando el viraje hacia el centro iniciado con la creación del Encuentro Progresista) y, por el otro, la adopción de un perfil de oposición nítido a las políticas de los sucesivos gobiernos (Yaffé, 2005: 161). Fue particularmente insistente, a lo largo de una década (entre 1995 y 2004), en el reclamo de "otra política económica". En ambas dimensiones, adoptó un perfil distinto al de Astori, que buscó, durante todos esos años, sin éxito, desplazarlo del liderazgo del FA y del papel de candidato a la presidencia.

Sin este preámbulo no es posible explicar cómo Mujica terminó, al final de gobierno de Vázquez, convirtiéndose en el candidato a la presidencia por el FA. Para asegurar su triunfo en la primera vuelta de la elección presidencial de octubre de 2004, Vázquez anunció que su ministro de Economía, en caso de ser electo presidente, sería Danilo Astori. Efectivamente, Astori asumió este cargo en marzo de 2005. Pero, para sorpresa de los partidos de oposición y de muchos frenteamplistas, funcionó, de hecho, como un "primer ministro". Desde el Ministerio de Economía y Finanzas, al frente de una extensa red de economistas de alto estándar académico, Astori logró imponer su visión en numerosas áreas de políticas públicas. Durante años, los frenteamplistas habían asistido a un duro enfrentamiento entre Vázquez y Astori. Y, en reiteradas ocasiones, se habían pronunciado a favor de los enfoques de Vázquez. De golpe, después de la elección primaria de junio de 2004, los adversarios se volvieron aliados. En suma, aunque la fracción de José Mujica fue la más votada en octubre, Danilo Astori fue el hombre más influyente en el entorno del presidente Vázquez. El apoyo a Mujica dentro del FA, y la demanda de su postulación como candidato a la presidencia, nació y se desarrolló, precisamente, en el espacio que el principal líder izquierdista, rápidamente devenido en presidente centrista, dejó libre. El cambio de escenario puede ilustrarse fácilmente con un par de ejemplos. En primer lugar, a comienzos de 2005 se mostraron reacios a facilitar la refinanciación de las deudas contraídas por los productores rurales con el principal banco público uruguayo. En segundo lugar, durante el 2006, ambos impulsaron la firma de un Tratado de Libre Comercio con EE.UU. Mujica se enfrentó abiertamente con Astori y Vázquez en lo relacionado con la refinanciación de las deudas de los productores y adoptó un perfil muy bajo en el debate acerca de la conveniencia de un TLC. Mientras el presidente y su ministro preferido chocaban contra la tradición, la imagen de Mujica se fortalecía ${ }^{17}$ y se comenzaba a instalar en la opinión pública, paulatinamente, que el ex guerrillero, a pesar de su pasado y de su tan peculiar estilo de comunicación, podía ser uno de los aspirantes a la sucesión de Vázquez. ${ }^{18}$

A principios de 2008 Vázquez excluyó a Mujica del gabinete, en el contexto de una reestructura general de su elenco de gobierno y empezó a dejar saber, a través de distintos canales, que, en su opinión, la mejor fórmula presidencial del FA era "Astori-Mujica", "en ese orden" (es decir, con Astori como candidato a la presidencia). La mayoría de las fracciones del

17 Desde mediados de 2006 en adelante, el "saldo neto simpatía-antipatía" de Mujica superó al de Astori.

18 Una clara mayoría de los frenteamplistas hubiera preferido que Tabaré Vázquez fuera, nuevamente, candidato a la presidencia en 2009. En Uruguay no existe la regla de la reelección inmediata. Excluyendo del "menú" al presidente, a fines de 2005 apenas $8 \%$ de los frenteamplistas mencionaban espontáneamente a Mujica como su candidato a la presidencia preferido. Un año después, esta cifra trepó al 30\%. Nadie había mencionado públicamente esta posibilidad. La preferencia por Astori en 2005 era del $23 \%$ y pasó al 31\%. Ver: http: / /www. gruporadar.com.uy/opinion/2006/17-11-06.pdf. Visitado por última vez el 7-02-2011. 
FA acompañaron la iniciativa del presidente. Pero Mujica pactó con los comunistas (que siguen predominando en la militancia de base) y fue proclamado candidato a la presidencia por el Congreso del FA (diciembre 2008). De todos modos, el Congreso habilitó que otros candidatos se presentaran a la elección primaria de junio (Danilo Astori, Marcos Carámbula y Enrique Rubio). En enero de 2009, Rubio declinó su candidatura y su fracción decidió acompañar la candidatura de Carámbula. La competencia terminó con una clara victoria el ex guerrillero: $52 \%$ para Mujica, $40 \%$ para Astori, $8 \%$ para Carámbula.

El MLN-T no fue un obstáculo para Mujica. Como siempre, siguió habiendo dentro la organización visiones diferentes respecto a qué podía realmente esperarse del camino electoral. A comienzos del gobierno de Vázquez se instaló un fuerte debate dentro del MLN-T sobre la posición a adoptar. Mujica logró administrar la tensión. Pero Fernández Huidobro, un par de años después, optó por renunciar al MLN-T. ${ }^{19}$ Más allá de tensiones y escisiones, fieles a su propia tradición política de admitir zonas de acción autónoma y permitir la realización simultánea de "experimentos" políticos diferentes, los menos entusiastas con la vía electoral (como Marenales) dejaron que Mujica hiciera bien su trabajo. Mientras tanto, al menos una parte de la estructura del MLN-T se dedicó a "vigilar" el comportamiento de gobernantes del FA (Labrousse, 2009: 295-297). Según diversas versiones de prensa, los tupamaros fueron actores fundamentales en algunas denuncias de corrupción contra gobernantes frenteamplistas. ${ }^{20}$

Cuadro 1: Evolución electoral del MLN-T (1971-2009)

\begin{tabular}{lccccccc}
\hline \multicolumn{1}{c}{ Elección } & 1971 & 1984 & 1989 & 1994 & 1999 & 2004 & 2009 \\
\hline $\begin{array}{l}\text { Etiqueta } \\
\text { electoral }^{*}\end{array}$ & Patria & IDI & MPP & MPP & MPP & E-609 & E-609 \\
Total de votos & 70.944 & 26.785 & 45.145 & 45.469 & 139.692 & 327.947 & 368.786 \\
$\%$ del FA & $23,3 \%$ & $6,7 \%$ & $10,8 \%$ & $7,3 \%$ & $16,3 \%$ & $29,1 \%$ & $33,6 \%$ \\
$\%$ del total & $4,3 \%$ & $1,4 \%$ & $2,3 \%$ & $2,2 \%$ & $4,7 \%$ & $15,1 \%$ & $16 \%$ \\
\hline
\end{tabular}

Fuente: Banco de Datos de Política y Relaciones Internacionales (FCS-Udelar).

Nota:

* Los tupamaros siempre votaron dentro de minicoaliciones con otros grupos de izquierda. La etiqueta electoral de la alianza que, en cada momento, los incluyó, fue cambiando con el tiempo. IDI: Izquierda Democrática Independiente. E-609: Espacio 609 (alianza entre el MPP, grupos pequeños y personalidades de izquierda).

19 Este importante documento puede leerse en: http://www.larepublica.com.uy/comunidad/270871-micontratapa-mas-triste. Visitado el 7-2-2011.

20 Algunas referencias a estas "actividades especiales" aparecieron en la prensa. Transcribo un pasaje de la nota del periodista Eduardo Barreneche en el diario El País (5/8/2007): “El MLN-Tupamaros dirige el funcionamiento de grupos 'especiales' que investigan a funcionarios, áreas de la administración para detectar actos de corrupción o situaciones que estiman relevantes en otras colectividades del Frente Amplio. (...). Estos grupos se dedican a recolectar información y a realizar este tipo de investigaciones, pero también realizan tareas especiales de seguridad, se encargan de las relaciones internacionales del MLN con fuerzas políticas similares del exterior de pasado guerrillero, e incluso investigan a integrantes de la propia organización. Las informaciones recabadas por estos grupos son elevadas a la dirección del MLN y, en algunos casos, a integrantes del Movimiento de Participación Popular, a otras fuerzas políticas de la coalición y al propio presidente Tabaré Vázquez". Ver: http://www.elpais.com.uy/07/08/05/pnacio_295921.asp. Visitado el 7-02-2011. 


\section{EL ARGUMENTO EN PERSPECTIVA COMPARADA: EL FRACASO DEL PARTIDO COMUNISTA DE URUGUAY}

El MLN-T es un caso notablemente exitoso de adaptación partidaria. Pasaron de las armas a las urnas, de guerrilleros derrotados a exitosos buscadores de votos, de presos políticos a gobernantes. Recorrieron este camino en relativamente poco tiempo. El éxito del MLN-T contrasta fuertemente con el fracaso del Partido Comunista de Uruguay.

Finalizado el régimen autoritario, el prestigio de los comunistas había aumentado significativamente. Esto se reflejó en un crecimiento organizativo y electoral explosivo. En la elección de 1989, la fracción más votada dentro del FA fue la liderada por los comunistas. Sin embargo, poco después, al compás de la crisis del campo socialista, el PCU entró en una crisis muy profunda.

En 1989, el MLN-T estaba a la izquierda del PCU. Una década después, se había colocado a su derecha. Mientras el MLN-T viraba hacia el centro y comenzaba a crecer, el PCU se desplomaba. En 1989 el PCU tenía cerca de 50.000 afiliados. Tres años después, dentro de la organización, quedaban apenas el 10\%. En 1989, la fracción constituida por los comunistas y sus aliados obtuvo cuatro senadores. Veinte años después, apenas obtuvo un senador. Mientras tanto, el MPP, que había obtenido apenas dos diputados en 1989, ahora tiene 25 diputados (el FA tiene 50) y seis senadores (el FA tiene 17).

Gráfico 1: Evolución electoral PCU y MLN-T (1971-2009)

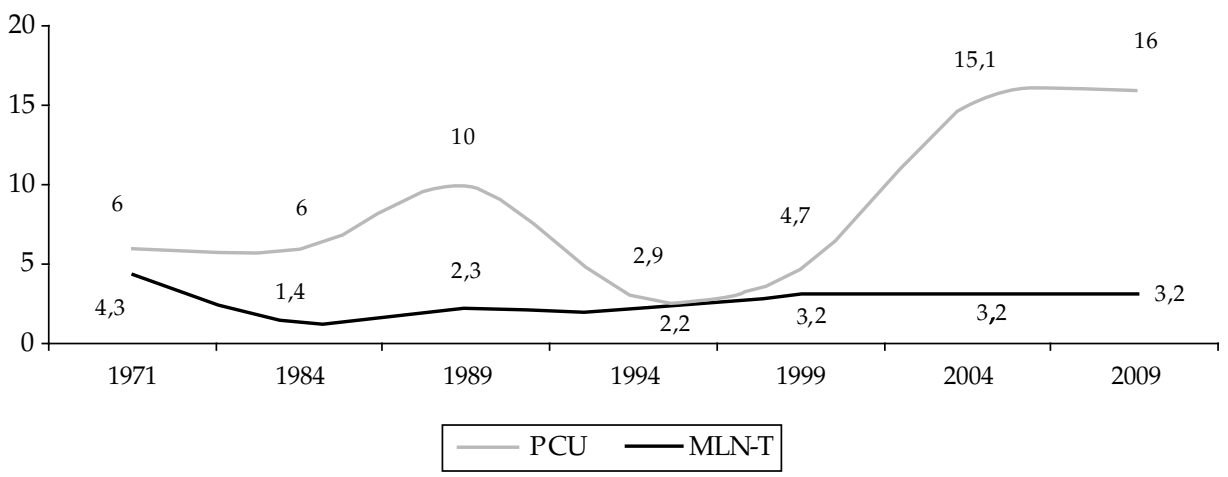

Fuente: Banco de Datos de Política y Relaciones Internacionales Facultad de Ciencias Sociales/ UdelaR. Datos son porcentajes sobre el total del electorado.

¿Qué es lo que explica que estas dos organizaciones políticas hayan tenido, entre 1985 y 2010, desempeños electorales tan diferentes? Las dos han estado sometidas a los mismos estímulos, tanto domésticos como externos. Ambas organizaciones pertenecen al FA: actúan, por tanto, en el mismo marco institucional y político, y enfrentan los mismos desafíos desde el punto de vista de la competencia electoral. Las dos organizaciones perdieron a su principal referente político en 1989: Raúl Sendic, el jefe tupamaro, murió 
en abril; Rodney Arismendi, el líder histórico de los comunistas en diciembre, poco después de ser electo senador.

No todas son semejanzas. Existen dos diferencias importantes. En primer lugar, las estructuras organizativas no son idénticas. El MLN-T tiene un patrón de reclutamiento mucho más selectivo que el PCU. El PCU, a su vez, admite menores niveles de descentralización en sus organizaciones y de debate interno que el MLN-T. En ambas organizaciones la dirección tiene niveles importantes de autonomía respecto a la militancia. Resumiendo, y retomando el enfoque de Kitschelt, los factores organizativos, cuando se los considera en conjunto, no deberían tener un efecto neto, desde el punto de vista de la adaptación partidaria, especialmente favorable al MLN-T. El patrón de reclutamiento del MLN-T no favorece la "innovación desde abajo" pero la descentralización y la apertura al debate interno sí. La autonomía de la dirección favorece, en las dos organizaciones, la “innovación desde arriba" (Kitschelt, 1994: 212-214).

En segundo lugar, existen diferencias significativas en el plano ideológico. Estas diferencias sí son importantes y ayudan a entender por qué comunistas y tupamaros han tenido trayectorias diferentes en el período analizado. Las dos son organizaciones socialistas, revolucionarias, antiimperialistas y latinoamericanistas. Pero tienen al menos tres grandes diferencias. En primer lugar, el PCU se caracterizó desde su fundación por una impactante fidelidad política al PCUS (De Giorgi, Lanza y Garcé, 2010). Mientras tanto, el MLN-T hizo de la independencia respecto a otros procesos revolucionarios un verdadero culto. En segundo lugar, mientras el PCU privilegió la homogeneidad ideológica (en torno al marxismo-leninismo), el MLN-T admitió la diversidad y propició el debate. En tercer lugar, las respectivas ideologías establecen relaciones diferentes entre teoría y práctica revolucionarias: para los comunistas primero está la teoría y luego la práctica; para los tupamaros la teoría revolucionaria va surgiendo de la práctica.

Estas diferencias de contenido ayudan a entender algunos momentos importantes de ambas trayectorias. Por ejemplo, el desplome de la URSS fue un golpe decisivo sobre el PCU porque, a lo largo de muchas décadas, los comunistas habían construido su fe en la "justeza de la línea" en lo que Arismendi denominada "el laboratorio de la praxis del siglo XX" que mostraba, según argumentaban sistemáticamente, un avance permanente del campo socialista. El MLN-T, en cambio, se vio mucho menos afectado por el desplome del campo socialista.

Pero, para entender por qué el MLN-T logró despegar electoralmente a partir de 1995 y el PCU no, hay que tomar en cuenta que la ideología tupamara es de un tipo distinto a la de los comunistas. El MLN-T logró crecer porque Mujica se convirtió en un aliado formidable del "giro al centro" liderado por Tabaré Vázquez. El contenido de la ideología tupamara (su historia de movimiento guerrillero, su profunda vocación revolucionaria y socialista) no le impidió moderar rápida y drásticamente el discurso. Mientras tanto, los comunistas siguieron aplicando el viejo libreto. Se negaron explícitamente a "adaptarse". ${ }^{21}$

21 Hace algunos años, el Secretario General del PCU pronunció esta frase esclarecedora: “En lo atinente a la línea política nosotros tenemos un punto de partida, que es la no aceptación de aquella concepción de la política 
Insistieron en jugar el papel de "memoria histórica" de la izquierda uruguaya. El MLN-T cambió de estrategia. El PCU no. Tomar en cuenta que ambas organizaciones tienen tipos de ideología diferentes es esencial para entender lo sucedido. Los tupamaros cambiaron porque siempre se distinguieron por su pragmatismo. Los comunistas uruguayos no cambiaron de estrategia porque son un ejemplo arquetípico de partido doctrinario. ${ }^{22}$ Este punto merece un párrafo aparte.

Nada de lo que han hecho los comunistas uruguayos ha sido improvisado. A mediados de la década del 50 el PCU definió con toda claridad su visión del proceso revolucionario uruguayo. Los lineamientos tácticos y estratégicos trazados en los congresos XVI (1955) y XVII (1958) constituyeron los pilares sobre los que se apoyó la actividad partidaria durante el período previo al golpe de Estado de junio de 1973. Con algunos matices, los comunistas uruguayos todavía hoy consideran que allí está trazada la ruta al socialismo en Uruguay. La estrategia y la táctica tenían cimientos de piedra. Una teoría así no se modifica todos los días. ${ }^{23}$ La práctica, en los comunistas, obedece a la teoría. Es la teoría, y no las circunstancias del contexto, la que guía la acción. A partir de 1989 se desplomó el campo socialista. En 1991 se disgregó la URSS. El PCU se sumergió en una crisis dramática. El entorno político cambió bruscamente. Pero el PCU no.

Los comunistas siguen pensando y haciendo lo mismo que hace dos o cuatro décadas. En cambio, hay una distancia realmente notable entre la práctica política del MLN-T durante la década del 60 y la de los que animan este segundo gobierno del FA. Los tupamaros pasaron de ser despreciar las instituciones "burguesas" a instalarse en ellas, de luchar por la "liberación nacional y el socialismo" a administrar el capitalismo uruguayo. Aunque muchos tupamaros a lo largo de este periplo abandonaron la organización, el MLN-T sigue existiendo y pesando en la política nacional. Los tupamaros no tienen ningún problema para justificar cómo es que han cambiado tanto. Argumentan, una y otra vez, que ellos no cambiaron, que cambiaron las circunstancias.

\section{CONCLUSIÓN}

La breve comparación entre el PCU y el MLN-T refuerza el argumento teórico presentado en este artículo. El pragmatismo que caracteriza a la matriz ideológica del MLN-T favoreció la adaptación partidaria mientras que la rigidez de la doctrina de los comunistas habría sido un obstáculo prácticamente insalvable.

como el "arte de lo posible", o en otras palabras, a la adaptación que conduce al oportunismo." Discurso de apertura del X Congreso Extraordinario Alberto Altesor a cargo de Eduardo Lorier, Congreso realizado del 20 al 22 de julio de 2007, p. 16.

22 A veces se define pragmatismo como ausencia de ideología.

23 Repasemos un ejemplo. Los comunistas uruguayos definieron, en esa misma época, mediados de los 50, que la vía más probable para la revolución en Uruguay (y toda América Latina) era la vía armada. Pero que Uruguay viviría, por un período prolongado, un "período de acumulación de fuerzas". En forma consistente con esta teoría organizaron, a partir de 1964, un aparato armado importante. Pero, a pesar de la radicalización del clima político uruguayo, de la creciente dialéctica de la violencia entre el movimiento guerrillero y el gobierno de la época, fieles a sus definiciones teóricas, optaron por no utilizarlo. Esta decisión, derivada de una elaboración teórica muy meditada, ni siquiera cambió en el momento del golpe de Estado. 
Al menos desde Panebianco en adelante existe cierto consenso en que la variable liderazgo importa. No hay adaptación sin líderes capaces de trazar e implementar cursos estratégicos inteligentes. A partir de otros autores, desde el propio Panebianco a Levitsky, también sabemos que no siempre la estructura organizativa favorece la emergencia de este tipo de liderazgos. El análisis del proceso de adaptación del MLN-T invita a seguir desarrollando la teoría en nuevas direcciones. Tal como sugirió Kitschelt, la tradición discursiva es una variable relevante en los procesos de adaptación partidaria. Parece claro que hay ideologías que favorecen más que otras los procesos de cambio y adaptación partidaria. No es casualidad que los partidos populistas (pragmáticos, oportunistas) tiendan a sobrevivir y los partidos marxista-leninistas (doctrinarios, dogmáticos) a languidecer. Mirado desde este ángulo, la capacidad de metamorfosis de organizaciones como el Partido Justicialista de Argentina no tiene mucho de misterioso. Si el argumento propuesto en este artículo fuera correcto, para explicar los desconcertantes virajes del justicialismo argentino en el plano de las posiciones sobre políticas públicas (ida y vuelta del estatismo al liberalismo), sin perjuicio de tomar en cuenta otras variables como las priorizadas por Levitsky (la combinación de una débil institucionalización con la persistencia de profundas raíces sociales), habría que empezar por enfatizar (o, mejor dicho, volver a subrayar) que la tradición ideológica peronista porta en su seno de modo explícito, y desde los primeros tiempos, un auténtico mandato imperativo a favor de la adaptación de la "doctrina" a las circunstancias históricas concretas. ${ }^{24}$ Siguiendo este razonamiento hasta sus últimas consecuencias, y parafraseando a Levitsky, ${ }^{25}$ podría afirmarse que la verdadera ventaja de los partidos populistas de masas de América Latina sobre otros, como los partidos comunistas, no está en el tipo de organización sino en las características de sus tradiciones ideológicas.

Evidentemente, no es razonable ni necesario llevar el hilo de la argumentación tan lejos. El objetivo de este artículo fue más modesto. A partir del estudio en profundidad de un caso, el del MLN-T, se procuró sugerir que para explicar los procesos de adaptación partidaria, además de tomar en cuenta los factores que la literatura ha venido privilegiando, puede ser necesario estudiar a fondo las características de las ideologías partidarias. El desafío para la teoría consiste en desentrañar las diferentes combinaciones de estas (y otras variables) que explican, en cada caso, éxitos y fracasos. Menem, resumió esta visión del modo siguiente: “El peronismo podrá haberse identificado, históricamente, con concepciones en torno al Estado y orientaciones político-económicas diametralmente opuestas a las que informan discursivamente el curso reformista adoptado. Pero al mismo tiempo es escuela del más vigoroso pragmatismo: "la única verdad es la realidad" (...)". En la misma línea, Malamud (2011) sostiene que el justicialismo, históricamente, ha sido más pragmático que ideológico y define a la política exterior durante los gobiernos de Néstor Kirchner y Cristina Fernández como "short term pragmatism".

25 Resumiendo su argumento dice Levitsky: “Hemos dicho que los partidos populistas de masas pueden tener una clara ventaja sobre los socialdemócratas, los comunistas y otros partidos de base obrera con respecto a sus posibilidades de adaptarse a los desafíos contemporáneos de la liberalización económica y la declinación de la clase obrera" (2005.324). 


\section{BIBLIOGRAFÍA}

Altman, David. 2010. "Uruguay 2009: Completing the Line-Up of Latin American Presidents". Electoral Studies 29 (3): 533-536.

Allison, Michael. E. 2010. "Grupos armados y partidos políticos. Un poco de ayuda por favor", en Alberto Martín Álvarez. La izquierda revolucionaria latinoamericana. México: Universidad de Colima, 131-156.

Arrarás, Astrid. 1998. "Armed Struggle, Political Learning, and Participation in Democracy: The Case of the Tupamaros (Uruguay)", Princeton University, PhD. Dissertation.

Blyth, Mark. 1997. "Any More Bright Ideas? The Ideational Turn of Comparative Political Economy (review article)", Comparative Politics 29 (2): 229-250.

Buquet, Daniel y Niki Johnson (coords.). 2010. Del cambio a la continuidad. Elecciones 2009-2010 en Uruguay. Montevideo: Editorial Fin de Siglo.

Caetano, Gerardo y José Rilla. 1995. "A la búsqueda de una historia perdida. Izquierda y Tradición: un problema y su versión en el Uruguay", en Gerardo Caetano, Javier Gallardo y José Rilla, La izquierda uruguaya: tradición, innovación y política. Montevideo: Ediciones Trilce, 11-51.

De Armas, Gustavo. 2005. “De la sociedad hiperintegrada al país fragmentado. Crónica del último tramo de un largo recorrido", en Gerardo Caetano (coord.). 20 años de democracia. Uruguay 1985-2005: miradas múltiples. Montevideo: Taurus, 269-303.

De Giorgi, Ana Laura, Adolfo Garcé y Federico Lanza. 2010. “Ideología y adaptación partidaria: El Partido Comunista de Uruguay y el colapso del campo socialista (1985-2009)". Ponencia en V Congreso Latinoamericano de Ciencia Política, Buenos Aires: ALACIP.

De Ipola, Emilio. 1987. "La difícil apuesta del peronismo democrático", en José Nun y Juan Carlos Portantiero (eds.). Ensayos sobre la transición democrática en Argentina. Buenos Aires: Punto Sur.

Garcé, Adolfo. 2006. Donde hubo fuego. El proceso de adaptación del MLN-Tupamaros a la legalidad y a la competencia electoral (1985-2004). Montevideo: Fin de Siglo.

Garcé, Adolfo. 2010. "Uruguay 2009: de Tabaré Vázquez a José Mujica", Anuario Político de América Latina, Revista de Ciencia Política 30 (2): 499-535.

Gatto, Hebert. 2004. El cielo por asalto. El Movimiento de Liberación Nacional (Tupamaros) y la izquierda uruguaya (1963-1972). Montevideo: Editorial Taurus-Santillana.

Kitschelt, Herbert. 1994. The Transformation of European Social Democracy. New York and Melbourne: Cambridge University Press.

Labrousse, Alain. 2009. Una historia de los tupamaros. De Sendic a Mujica. Montevideo: Editorial Fin de Siglo.

Lanzaro, Jorge. 2004. "La izquierda se acerca a los uruguayos y los uruguayos se acercan a la izquierda. Claves de desarrollo del Frente Amplio", en Jorge Lanzaro (coord.). La izquierda uruguaya entre la oposición y el gobierno. Montevideo: Editorial Fin de Siglo, 13-107.

Leicht, Federico. 2007. Cero a la izquierda. Una biografía de Jorge Zabalza. Montevideo: Ediciones Letra Eñe.

Lessa, Alfonso. 2004. La revolución imposible. Los tupamaros y el fracaso de la vía armada en el Uruguay del siglo XX. Montevideo: Editorial Fin de Siglo.

Levitsky, Steven. 2001. "An Organized Disorganization: Informal Organization and the Persistence of Local Party Structures in Argentine Peronism". Journal of Latin American Studies 33 (1): 40-43.

Levitsky, Steven. 2005. La transformación del justicialismo. Del partido sindical al partido clientelista. 1983-1999. Buenos Aires: Siglo XXI-Editorial Sudamericana.

Malamud, Andrés. 2001. "Argentine Foreign Policy Under The Kirchners: Ideological, Pragmatic Or Simply Peronist?", Gian Luca Gardini and Peter Lambert (eds.), Latin American Foreign Policies. Between Ideology and Pragmatism. Palgrave Macmillan, forthcoming.

Martí i Puig, Salvador. 2010. "The Adaptation of the FSLN: Daniel Ortega's Leadership and Democracy in Nicaragua". Latin American Politics and Society 52 (4): 80-106.

Palermo, Vicente. 1992. "El menemismo, ¿perdurará?" Buenos Aires: Centro de Estudios de Estado y Sociedad - Barcelona: Departament de Ciència Política i de Dret Públic, Universitat Autònoma de Barcelona. 
Panebianco, Angelo. 1995. Modelos de Partido. Madrid: Alianza Editorial.

Panizza, Francisco. 1990. Uruguay: Batllismo y después. Pacheco, militares y tupamaros en la crisis del Uruguay batllista, Montevideo: Ediciones de la Banda Oriental.

Rey Tristán, Eduardo. 2005. La izquierda revolucionaria uruguaya, 1955-1973. Sevilla: Universidad de Sevilla-CSIC.

Samuels, David. 2004. "From Socialism to Social Democracy. Party Organization and the Transformation of the Workers Party in Brazil". Comparative Political Studies 20 (10): 1-26.

Santiuste Cué, Salvador. 2001. “La «incompleta» transformación del FSLN". América Latina Hoy 27: 89122.

Schmidt, Vivien A. 2008. "Discursive Institutionalism: The Explanatory Power of Ideas and Discourse". Annual Review of Political Science 11: 303-326.

Yaffé, Jaime. 2005. Al centro y adentro: la renovación de la izquierda y el triunfo del Frente Amplio en Uruguay. Montevideo: Ediciones Linardi y Risso.

Adolfo Garcé se desempeña como Profesor Agregado en Régimen de Dedicación Total en el Instituto de Ciencia Política de la Facultad de Ciencias Sociales, Universidad de la República (UdelaR) y como Profesor Adjunto en la Cátedra de Ciencia Política de la Facultad de Ciencias Económicas y de Administración (UdelaR). Es candidato a Doctor en Ciencia Política e investigador Nivel I en el Sistema Nacional de Investigadores.

E-mail: agarce@fcs.edu.uy 
